\title{
Pyoderma gangrenosum mimicking inflammatory breast cancer
}

\author{
Agnieszka Białecka', Kaja Męcińska-Jundziłł¹, Urszula Adamska', Magdalena Górecka-Sokołowska ${ }^{1}$, \\ Artur Czaplewski ${ }^{1}$, Aleksandra Grzanka1, Marcin Białecki², Katarzyna Nadolska², Rafał Czajkowski ${ }^{1}$
}

${ }^{1}$ Chair of Dermatology, Sexually Transmitted Diseases and Immunodermatology, Faculty of Medicine in Bydgoszcz, Nicolaus Copernicus University in Torun, Poland

${ }^{2}$ Department of Radiology and Diagnostics Imaging, Collegium Medicum in Bydgoszcz, Nicolaus Copernicus University in Torun, Poland Adv Dermatol Allergol 2017; XXXIV (5): 501-503 DOI: https://doi.org/10.5114/pdia.2016.62464

Pyoderma gangrenosum (PG) is a rare inflammatory dermatosis characterised by painful ulcerations. The onset of the condition may be sudden and its course rapid. Moreover, early diagnosis and application of appropriate treatment are important as the disease can lead to numerous complications. We present a case of a female patient with diagnosed PG mimicking inflammatory breast cancer.

A 61-year-old patient was admitted to the Department of Dermatology to diagnose and treat skin lesions manifesting as ulceration of the left breast and erythema localised on the trunk with concomitant extensive sclerosis of the subcutaneous tissue (Figure $1 \mathrm{~A}$ ). The patient suffered from internal disorders, including type 2 diabetes, arterial hypertension, hypothyroidism and obesity. Before admission to the Department of Dermatology the patient was hospitalised in the Internal Ward due to fever and persisting weakness. During the hospitalization, large, flaccid bullae with accompanying erythema occurred on the skin of the left breast, followed by corresponding skin lesions on the trunk. Wound swabs were collected and three types of bacteria were cultured (Staphylococcus spp., coagulase neg., Corynebacterium sp., Acinetobacter baumannii). The patient was isolated and targeted intravenous antibiotic therapy was initiated. The intensive treatment did not lead to clinical improvement, the ulceration within the left breast occurred and the patient was referred to the Department of Dermatology. During the hospitalization we observed increased blood inflammatory markers (C-reactive protein $58.80 \mathrm{mg} / \mathrm{dl}$, erythrocyte sedimentation rate after $1 \mathrm{~h} 85 \mathrm{~mm}$, total protein value $7.7 \mathrm{~g} / \mathrm{dl}$ ). Serum protein electrophoresis demonstrated increased levels of $\alpha$ globulins, $\gamma$ globulins and hypoalbuminemia. Blood cell count demonstrated a slightly increased platelets level, up to
$386 \times 10^{3} / \mu \mathrm{l}$, monocytosis and eosinopaenia. Moreover, antistreptolysin was $200 \mathrm{lU} / \mathrm{ml}$, anti-nuclear antibodies (ANA) were positive at a titre of $1: 320$ with a cytoplasmic type of luminescence, rheumatoid factor was $8 \mathrm{lU} / \mathrm{ml}$. Gram-negative bacilli were cultured from the swab of the left breast ulceration and assessed as a contamination. Ultrasound examination of the left breast revealed an apnoeic area with hyperechogenic streaks in the inferior internal quadrant suggesting an organising liquid area. Inflammatory breast cancer could not be excluded in the radiologist's opinion (Breast Imaging-Reporting and Data System USG $4 b$ - intermediate suspicion of malignancy, indicated urgent further diagnostics). Test results and clinical presentation led to the hypothesis of erysipelas, possibly constituting a paraneoplastic syndrome. Two skin biopsies were collected from the edge of ulceration and from the erythema of the trunk. Histopathology examination of the first lesion showed a non-characteristic presentation and the second examination showed presentation resembling scleroderma. The patient was discharged home in overall good condition with a recommendation of urgent oncology diagnostics in the Clinic of Breast Diseases.

Further imaging diagnostics were performed in the Oncology Centre. Ultrasound examination and magnetic resonance imaging of breasts suggested inflammatory infiltration. However, inflammatory cancer could not be excluded. Fine needle aspiration biopsy (FNAB) was performed under ultrasound guidance. Smears demonstrated presence of acellular serous content. A fragment of tissue was collected for histopathology re-assessment. The examination indicated non-specific inflammatory granulation.

Five months later the patient was re-admitted to the Department of Dermatology. Ulceration of the left breast

Address for correspondence: Agnieszka Białecka MD, Chair of Dermatology, Sexually Transmitted Diseases and Immunodermatology, Faculty of Medicine, Nicolaus Copernicus University, 9 Skłodowskiej-Curie St, 85-094 Bydgoszcz, Poland, phone: +48 696557 558, +48 535854 568, fax: +48 5258540 18, e-mail: agnieszka_bialecka@wp.pl Received: 2.08.2016, accepted: 5.09.2016. 

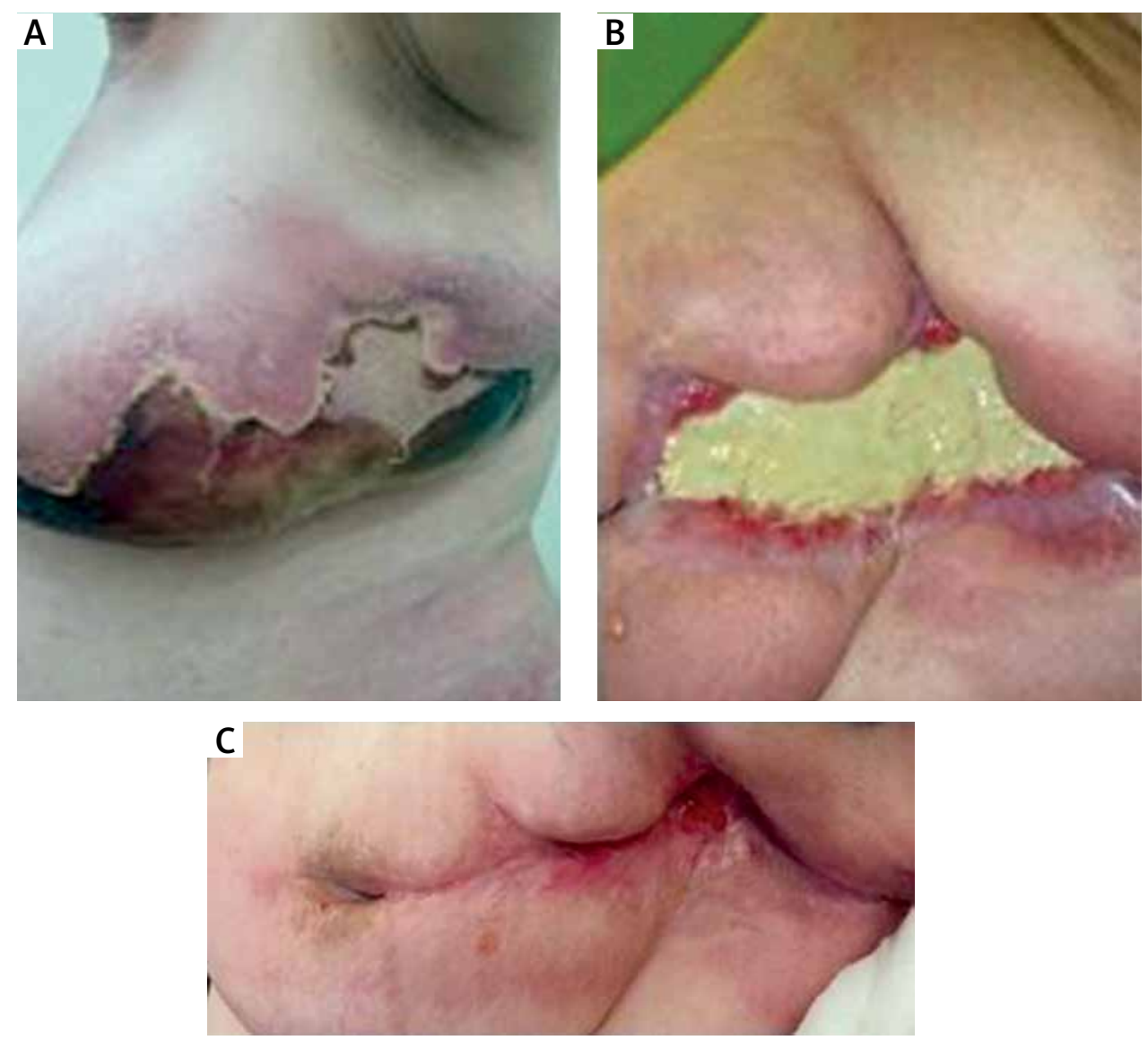

Figure 1. A - Extensive ulceration of the breast during the first hospitalization. B - Ulceration with a visible undermined violaceous area during the second hospitalization. $\mathbf{C}$ - Ulceration healing several months after treatment with cyclosporine $\mathrm{A}$

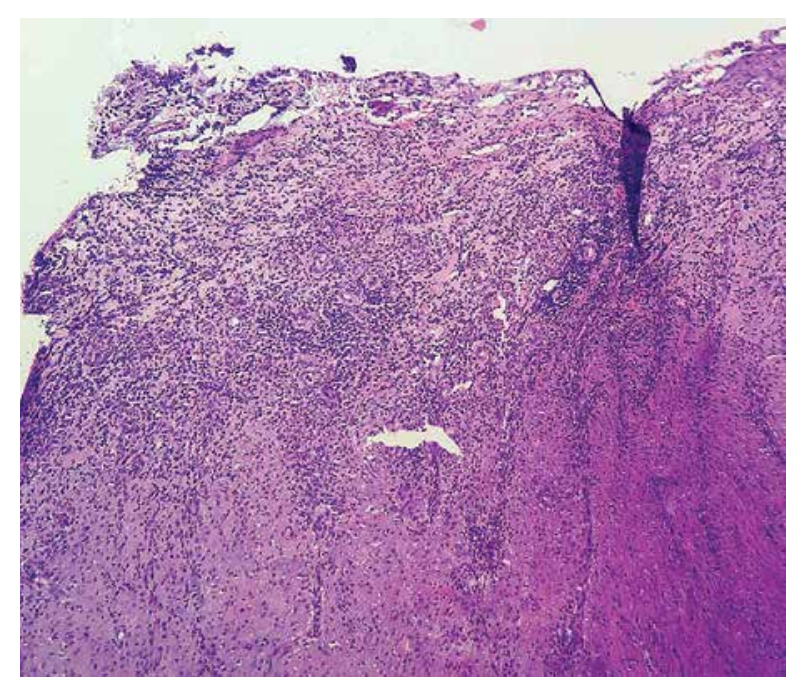

Figure 2. Biopsy specimen from the edge of the breast ulceration showing inflammatory infiltration of neutrophils, but also present lymphocytes, histiocytes, plasma cells and fibroblasts; in the dermis, small and medium-sized vessels inflamed with thickened walls was less extensive. However, an undermined violaceous area was visible around the skin lesion (Figure 1 B). Laboratory tests (morphology, biochemistry) revealed no significant abnormalities. Results of additional tests such as thyroid profile, anti-HIV and anti-HCV antibodies, HBs antigen, protein electrophoresis were unremarkable. Acinetobacter baumannii was cultured from the ulceration swab but considering normal levels of inflammatory markers the systemic antibiotic therapy was not initiated. Another skin biopsy was collected. Histopathologic evaluation suggested PG (Figure 2). Based on the clinical presentation and the result of the histopathological examination, pyoderma gangrenosum was eventually diagnosed, after exclusion of diseases that could be the cause of PG. Treatment with cyclosporine A at the dose of $2.7 \mathrm{mg} / \mathrm{kg}$ bw/day was initiated. The therapy was continued in the Outpatient Dermatology Clinic and gradual healing of ulceration was observed (Figure $1 \mathrm{C}$ ).

Pyoderma gangrenosum is a rare neutrophilic dermatosis characterised by development of painful ulcerations with a visible undermined violaceous edge usually localised on the lower limbs. Clinically the disease starts with 
pustules, vesicles or papules which rapidly transform into necrotic ulceration [1-3]. Its aetiology remains obscure. Pyoderma gangrenosum may develop as an isolated disease, but commonly, in 50-70\% of cases it coexists with other conditions, including inflammatory bowel diseases, hepatic diseases, rheumatoid arthritis and haematological disorders [1]. It may also coexist with malignant tumours, including breast, lung, large intestine or prostate gland cancers [1-4]. Malignant lesions should be considered particularly in patients with no concomitant characteristic diseases for PG, in patients with a medical history of cancer and in patients with recurrent PG-type lesions of rapid onset [5].

Pyoderma gangrenosum lesions localised within mammary glands are relatively rare. Majority of reported cases occurred in patients after surgical intervention [6-10], what is associated with a well-known pathergy phenomenon occurring in PG. The lesions develop at the site of skin damage and extend from that site onwards. In the case of our patient, development of ulceration was not associated with any previous skin injury, what hindered initial and final diagnosis.

To diagnose PG, other diseases that can cause ulcerations must be excluded [11]. In the case of our patient, we considered not only the diagnosis of inflammatory breast cancer, but also necrotizing fasciitis, severe cellulitis, erysipelas or other acute bacterial infections of the breast. The final diagnosis was based on clinical presentation and the result of the histopathological examination.

The treatment of PG consists of suppression of the inflammatory process, treatment of other coexisting diseases and reduction of pain. Immunosuppressants, including glucocorticosteroids, cyclosporine A, azathioprine and mycophenolate mofetil are effective. Other drugs, such as tumor necrosis factor $\alpha$ (TNF- $\alpha$ ) antagonists and intravenous immunoglobulins (IVIG) are far less commonly used [12]. Various types of treatment have also been reported like sulphasalazine, dapsone, thalidomide, colchicine, minocycline, tacrolimus, clofazimine, methotrexate or cyclophosphamide $[4,13]$. Systemic antibiotic therapy is often used in order to prevent secondary bacterial infections. Tetracyclines, vancomycin, rifampicin and mezlocillin may lead to improvement due to their anti-inflammatory effect [13]. There are currently no universal recommendations regarding PG therapy. Usually, the condition is treated according to experience of an individual therapeutic centre. There are reports indicating that treatment with systemic glucocorticosteroids (0.5-1.0 mg methylprednisolone/kg bw/day) and/or cyclosporine A ( $5 \mathrm{mg} / \mathrm{kg}$ bw/day) is recommended as the first-choice therapy of PG leading to rapid remission [14]. In our case, cyclosporine A in monotherapy was initiated concerning adverse effects of glucocorticosteroids as the patient suffered from type 2 diabetes and obesity. Ther- apy with cyclosporine A at the dose of $2.7 \mathrm{mg} / \mathrm{kg}$ bw/day resulted in gradual remission of skin lesions.

Pyoderma gangrenosum remains a serious diagnostic and therapeutic challenge for dermatologists. Exclusion of the possible ongoing neoplastic process that might coexist with the disease is necessary before the final diagnosis and introduction of immunosuppressive treatment.

\section{Conflict of interest}

The authors declare no conflict of interest.

\section{References}

1. Wollina U. Pyoderma gangrenosum - a review. Orphanet J Rare Dis 2007; 2: 19.

2. Adışen E, Erduran F, Gürer MA. Pyoderma gangrenosum: a report of 27 patients. Int I Low Extrem Wounds 2016; 15: $148-54$.

3. Romańska-Gocka K, Cieścińska C, Zegarska B, et al. Pyoderma gangrenosum with monoclonal IgA gammopathy and pulmonary tuberculosis. Illustrative case and review. Postep Derm Allergol 2015; 32: 137-41.

4. Ruocco E, Sangiuliano S, Gravina AG, et al. Pyoderma gangrenosum: an updated review. J Eur Acad Dermatol Venereol 2009; 23: 1008-17.

5. Shahi V, Wetter DA. Pyoderma gangrenosum associated with solid organ malignancies. Int J Dermatol 2015; 54: 351-7.

6. Juhász ML, Maman DY, Levin JM, et al. Pyoderma gangrenosum of the breast in a patient with a history of silicone augmentation mastopexy and suction-assisted lipectomy of the trunk. JAAD Case Rep 2015; 1: 329-32.

7. Davis MD, Alexander JL, Prawer SE. Pyoderma gangrenosum of the breasts precipitated by breast surgery. J Am Acad Dermatol 2006; 55: 317-20.

8. Duval A, Boissel N, Servant JM, et al. Pyoderma gangrenosum of the breast: a diagnosis not to be missed. J Plast Reconstr Aesthet Surg 2011; 64: 17-20.

9. Rietjens M, Cuccia G, Brenelli F, et al. A pyoderma gangrenosum following breast reconstruction: a rare cause of skin necrosis. Breast J 2010; 16: 200-2.

10. Horner B, El-Muttardi N, Mercer D. Pyoderma gangrenosum complicating bilateral breast reduction. Br J Plast Surg 2004; 57: 679-81.

11. Su WP, Davis MD, Weenig RH, et al. Pyoderma gangrenosum: clinicopathologic correlation and proposed diagnostic criteria. Int J Dermatol 2004; 43: 790-800.

12. Herberger K, Dissemond J, Hohaus K, et al. Treatment of pyoderma gangrenosum - retrospective multicenter analysis of 121 patients. Br J Dermatol 2016; 175: 1070-2.

13. Gameiro A, Pereira N, Cardoso JC, et al. Pyoderma gangrenosum: challenges and solutions. Clin Cosmet Investig Dermatol 2015; 8: 285-93.

14. Reichrath J, Bens G, Bonowitz A, et al. Treatment recommendations for pyoderma gangrenosum: an evidence-based review of the literature based on more than 350 patients. J Am Acad Dermatol 2005; 53: 273-83. 